\title{
Wireless Telemetry System for Real-Time Estimation of Ship Air Wakes with UAVs
}

\author{
Anil Kumar ${ }^{\text {a }}$, Pinhas Ben-Tzvi ${ }^{* a}$, Wael Saab ${ }^{\text {a }}$, Murray R. Snyder ${ }^{\text {b }}$ \\ ${ }^{a}$ Department of Mechanical Engineering, \\ Virginia Tech, Blacksburg VA 24060, USA \\ ${ }^{\mathrm{b}}$ Aerospace Engineering Department, US Naval Academy, Annapolis MD 21402, USA \\ *Corresponding Author. Email: bentzvi@,vt.edu
}

\begin{abstract}
This paper presents a wireless instrumentation system developed for real-time estimation of air turbulence patterns arising from the interaction of wind with any structure under consideration, which is an important study in the aerospace industry. In particular, this paper focuses on the application of the proposed system in a naval research problem for off-board measurement of ship air wake patterns using an instrumented radio controlled (RC) helicopter. We propose use of an Inertial Measurement Unit (IMU) as a sensor to measure air wake in the form of induced vibrations on the helicopter while it maneuvers through regions of active air wake. The proposed system makes use of Back Propagation Neural Networks to compensate for the vibrational noise contributed by pilot inputs. The instrumentation system was integrated and tested on a modified training vessel in the Chesapeake Bay, which provided a wide range of wind conditions.
\end{abstract}

Keywords - Air Turbulence Estimation, Artificial Neural Networks, RC Helicopter, Ship Air Wake, Wireless Instrumentation, Telemetry 


\section{INTRODUCTION}

The aerospace industry has always expressed a need for new technological advancements and techniques capable of redefining design and operational processes, which facilitate the progress of exploration and science. Considerable research has been devoted to monitoring structural vibrations [1]. Yet, determining air turbulences (often the main cause of structural vibrations and risk to aerial vehicles) is a challenging task which researchers continually explore and try to understand.

The most commonly used device for measuring air turbulence is the Hot Wire Anemometer (HWA) which relates the resistance of a wire, held at a constant temperature, to the speed of airflow at a single point in space. Researchers have used HWA for testing wind tunnel streams, model testing, and investigating turbulence problems in boundary layers, wakes of jets and channels [2]. Sonic Anemometers offer additional capabilities of both airflow speed and direction using ultra sonic sound waves; measurements are taken with fine temporal resolution making them very well suited for turbulence measurements [3]. These delicate sensors are expensive, require constant recalibration, and sturdy platforms during data collection since motions of the probe result in additional air flow velocity measurements. In order to obtain information about air turbulence in a wide area, a large number of these sensors should be mounted on sturdy rigging. In some cases riggings cannot be installed to cover an entire area of interest. For example, measuring off deck ship air wake activity for developing safe launch and recovery envelops of aerial vehicles [4]. Alternatively, a single sensor can be placed on a beam and swept about the entire area. This method suffers from deflections of long beams due to pitch/roll motion of the ship and causes random fluctuations in wind measurements and produces false information about the air turbulence. 
Computational Fluid Dynamics is a popular method of generating extrapolated velocity fields offering valuable insight to turbulent vortices. The computer-aided designs of the model and environment are often simplified to reduce computation loads making computational methods insufficiently validated for systems with complex structures. In addition, these simulations often need experimental validation using anemometer sensors, which have their respective drawbacks [5-9].

There is a need to develop a new technique that accurately detects turbulence patterns in large, open spaces. A new means of turbulence data collection in the Aerospace Industry will bring about enhanced flight research capabilities, and improvements in both aerial vehicle design and operational safety analysis. Our proposed system named Wireless Telemetry System (WTS) utilizes an RC helicopter flying in an area of interest with active turbulence regions to estimate and detect turbulence patterns in real-time. Normally machine learning techniques are used as optimum controller in robotic systems [10-12]. The proposed system utilizes trained neural networks that act as filters to eliminate pilot induced vibrations from recorded vibrational data. Results are mapped out visually on a Graphical User Interface (GUI) to better understand turbulence patterns. Experimental results and validation of our instrumentation system is presented through a case study performed to detect turbulence patterns and monitor helicopter interactions with ship air wakes created by air flowing over the ship superstructure.

\section{PROPOSED SYSTEM}

Air wake is turbulence originating from pressure gradients that result in violent wind gusts. The motivation of the WTS comes from observing an RC helicopter flying through active air wake zones. During flight, it experiences differential airflow velocities that cause tilting of the aircraft since the center of mass and effective geometric center do not coincide. We can infer that 
monitoring angular velocity patterns of a helicopter will provide a good description of air wake patterns. Thus, our proposed system detects air wake patterns in wide open areas by monitoring the RC helicopter angular rates using a Gyroscope. Fig. 1 shows tilting of an RC helicopter with angular velocity $\omega$ as a result of differential wind velocity (V1>V2).

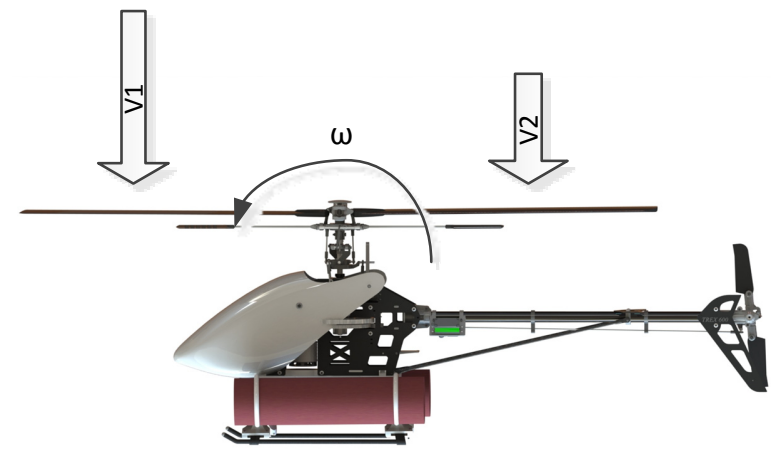

Figure 1 Interaction of air wake with helicopter resulting in tilting

It is important to note that the presence of air wake is not the only cause of angular velocity changes of the helicopter during flight. Cyclic pilot inputs change pitch angles of the rotor blade and result in differential thrust and consequently force moment on the helicopter fuselage. Thus, if this angular acceleration due to pilot inputs is known and the data sampling rate remains constant, the angular rate due to pilot input at any point in time can be estimated from the previous angular rate measurement. In general, helicopter is as a non-linear multiple input and multiple output system and can be modelled as follows:

$$
x(t)=f(x(t-1), u(t-1))
$$

where $x(t)$ represents the system state variables, such as angular and linear positions/velocities at any time $t$, and $u$ represent external inputs to the system. These external inputs constitute both pilot inputs and ship air wake disturbances. Angular rates of the RC helicopters measured at any time from the Gyroscope mainly depend on parameters related to the previous state variables 
[13-14] (i.e., linear velocities, angular rates, rotor speed and altitude, etc.) and external inputs as shown in (1). During air wake measurements, if flight parameters such as rotor speed, altitude and linear velocities are not drastically changed, then it is safe to assume that the angular rates mainly depend on pilot inputs, ship air wake and the previous measurements of angular rates. The major components of tilting and oscillations are caused by pilot input responses. The proposed system utilizes machine-learning techniques to estimate and compensate for dynamics arising from pilot inputs in order to isolate air wake induced effects on the aircraft.

The WTS is composed of three components viz. transmitter module, receiver module and transmitter carrier (RC Helicopter). Both the transmitter module and the receiver module are similar custom-made instrumentation boards mounted with sensors like IMU, GPS, Thermometer, and Barometer sensors. The transmitter module measures the dynamics of the helicopter and sends the data to the receiver module over $\mathrm{Xbee}{ }^{\mathrm{TM}}$ long range RF transceiver. The receiver module measures the dynamics of the boat and sends the measured data along with the transmitter module's data to a computer via a USB channel for real time processing. The RC helicopter was selected to carry the transmitter because of its low cost, high maneuverability and hovering capabilities.

The WTS was used to detect ship air wake turbulence in the aft of a cruising US Naval Academy's YP676 for estimation of safe launch and recovery envelopes. The data measured by the transmitting module on helicopter was routed wirelessly to the receiver module connected to a laptop located on the ship and displayed on a GUI. During data collection, the YP craft master maintained consistent speed and wind conditions based on the reference anemometer while the pilot swept the RC helicopter back and forth behind the ship. An experienced pilot maneuvered the RC helicopter (with the transmitting module) in a wave-like pattern at constant height in the 
aft of the boat. During helicopter maneuvers, the pilot responded to wind gusts in order to keep the helicopter stable. Such responses introduce tilting/vibrations in the IMU data and are highly subjective in nature.

In order to accurately measure actual air wake interactions, pilot induced dynamic inputs must be removed from the IMU data. The RC helicopters require five dimensional pilot input in the form of PWM signals. Three of which control swash plate kinematics and the remaining two control the tail rotor pitch and rotor speed. All five pilot input channels contribute to helicopter's attitude. Since angular velocity measurements are a vector sum of external disturbances and pilot induced dynamics, external air wake disturbances can be obtained by subtracting predicted IMU readings from actual readings. Pilot input from the radio transmitter (Remote Controller) controls the helicopter's attitude and is sent in 'one-to-all' broadcasting mode. Thus multiple RF receivers can receive the same signal from a single transmitter. Therefore, an additional RC receiver was used in the receiver module to read the pilot inputs sent by the transmitter. WTS uses Back Propagation Neural Networks (BPNN) to find mapping of IMU reading with pilot input signals. The air wake intensity was estimated by subtracting the BPNN generated IMU readings from the actual IMU readings in real-time.

The real-time location of the helicopter was estimated in the boat's frame of reference using GPSs in the receiver and the transmitter modules and a real-time trajectory of helicopter was obtained. The helicopter trajectory, IMU vibrational components, and pilot inputs are displayed and recorded in real time through GUI software developed using NI LabVIEW ${ }^{\mathrm{TM}}$. By fusing sensor data, possible locations of sharp gradients in the air velocities can be mapped relative to the ship representing helicopter/air wake interaction patterns (accurate within one rotor diameter 
of the helicopter). Fig. 2 shows the architecture of WTS proposed to measure ship air wake patterns.

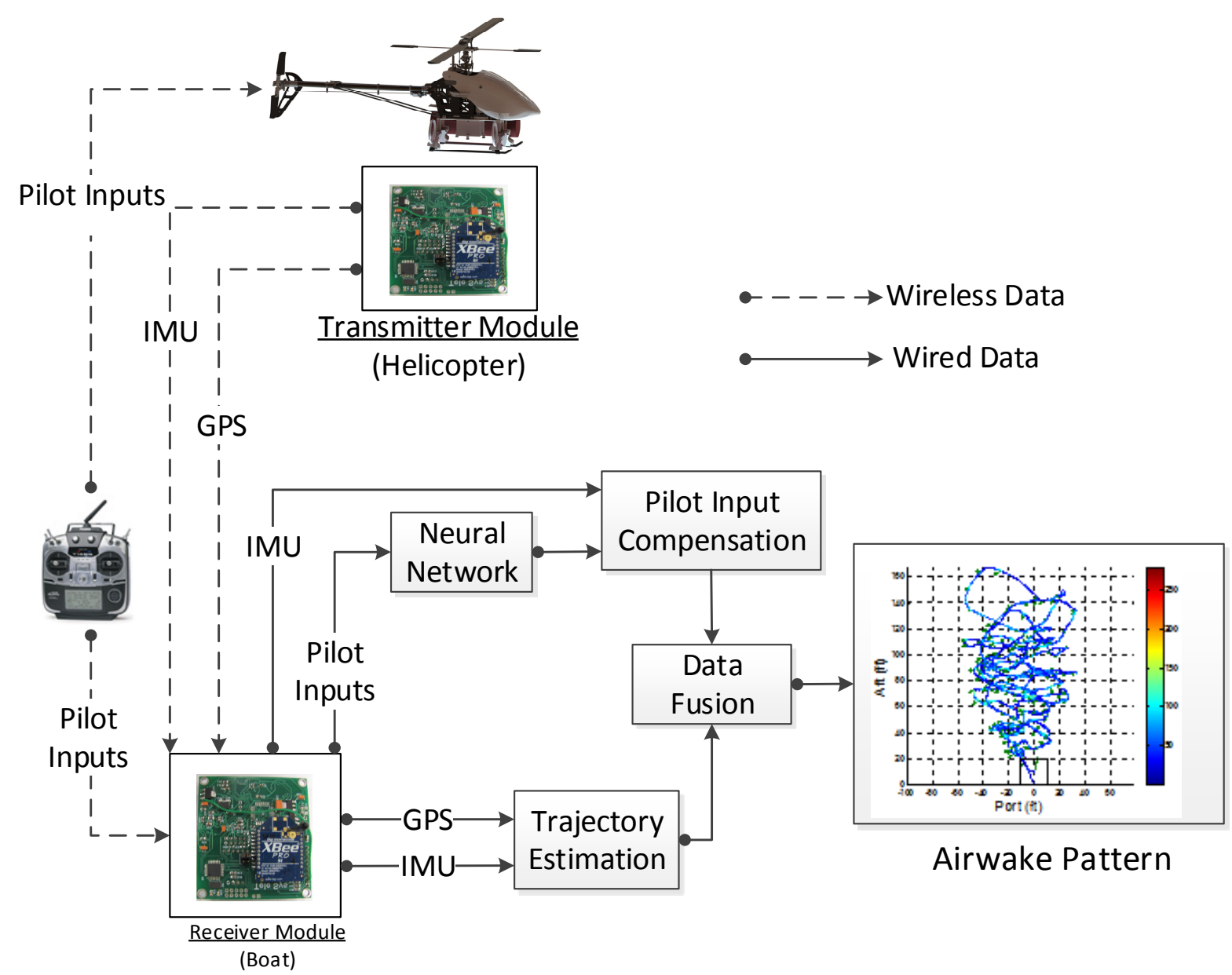

Figure 2 Schematics of the wireless telemetry system

\section{HARDWARE DETAILS}

The WTS used three different off-the-shelf RC helicopters with rotor diameter of $1.3 \mathrm{~m} \mathrm{(4.5 \textrm {ft } )}$ to detect air wake patterns generated by the YP676 vessel. The YP676 vessel was equipped with an eight channel ultrasonic anemometer array for maintaining a consistent wind condition during flight tests. Both transmitter and receiver modules are battery powered, independent sensor boards based on ATmega128 and ATmega8 microcontrollers. To ensure interchangeability, both sensor modules were deliberately designed to be similar both in terms of hardware and software. 
Each of the sensor modules contains GPS, 6 axes IMU, thermometer, 3D magnetometer, and barometer sensors. The sensor boards are equipped with micro SD card data logger, Quad USB to UART IC chip, micro USB interface, XBee RF module, and Futaba ${ }^{\mathrm{TM}} 8$ channel $2.4 \mathrm{GHz}$ RC receiver interface. The task of the transmitter module is to read all the sensors, send all the data to the SD card over SPI bus and transmit the same data over XBee wireless link.

The receiver module connected to a computer via USB reads all of the onboard sensors, records the data on SD card, and sends the same to computer via one of the four virtual serial COM ports. The XBee link on the receiver module receives data from the transmitter module and directly sends data to the computer over another serial COM port. The microcontroller on the receiver module reads pilot inputs in the form PWM signals and sends these signals to a computer over a third serial COM port at an update rate of $45 \mathrm{~Hz}$. Therefore, the receiver module, over three serial ports, provides sensor data from the transmitter module and receiver module and pilot input data via USB link.

Since both sensor modules are interchangeable, the mode of operation of the module is selected by a jumper switch. Fig. 3 shows the connections schematics of the sensor modules of the WTS.

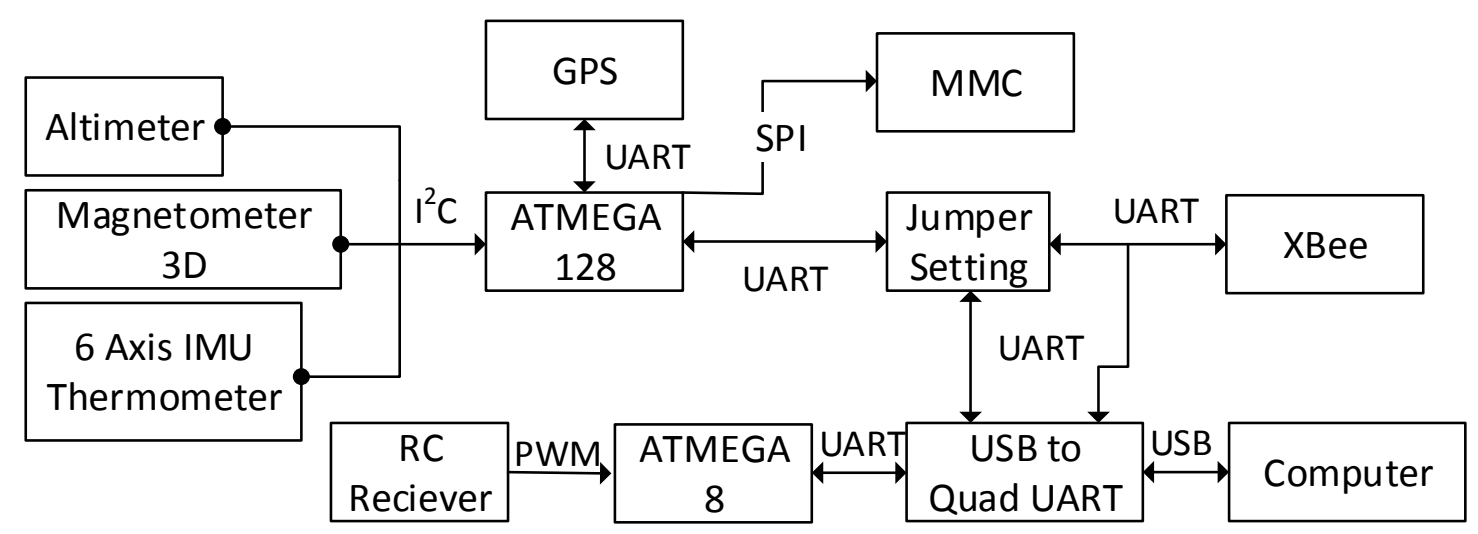

Figure 3 Hardware schematics of sensor modules 
The transmitter module and the receiver module were both rigidly fitted and oriented on the helicopter and the boat respectively using custom made 3D printed mountings. Rigid mounts were necessary in order to accurately capture angular velocity components of the helicopter while in flight due to the sensors high sensitivity. Fig. 4 shows the transmitter module fitted on the helicopter and the receiver module fitted on the ship using 3D printed mountings.
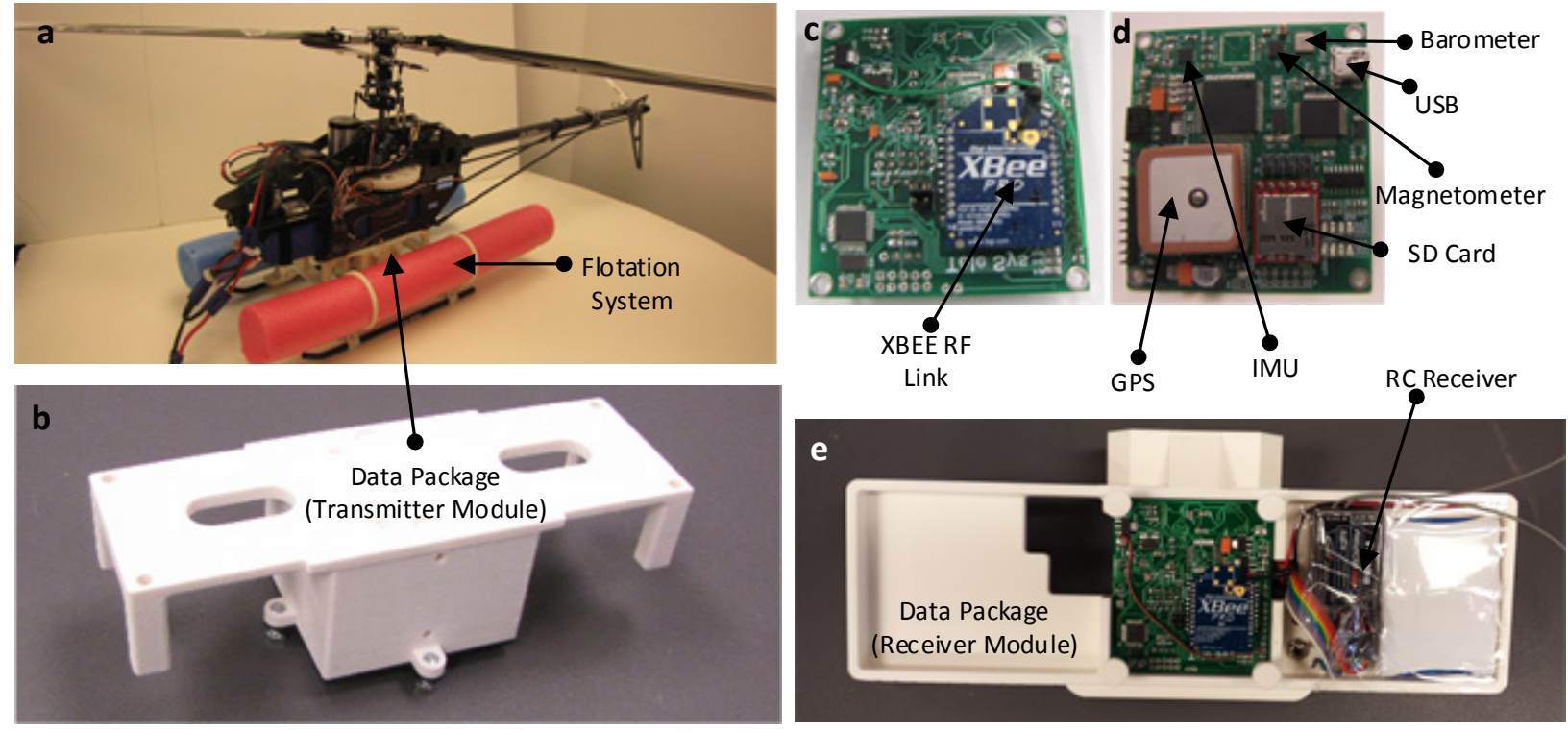

Figure 4 Hardware Setup: (a) Helicopter fitted with Data Package and Flotation system, (b) Data package with transmitter module inside, (c) Transmitter module (Top), (d) Transmitter module (Bottom), (e) Receiver module

\section{ESTIMATION OF PILOT INDUCED VIBRATIONS}

Artificial Neural Network (ANN) is one of the most studied [15-18] computational model in the field of machine learning and pattern recognition. Neural networks are widely used with wireless sensor networks [19-20]. Coarsely speaking, a neural network is an inter-connected network composed of processing units (neurons) where each neuron is a multi-input and single output system. Each neuron calculates the weighted sum of all inputs, subtracts a characteristic value (bias) from the sum and then applies a characteristic function to obtain the output of the neuron. The capacity of a neural network to modal complex data largely depends on the network 
topology and the characteristic function associated with each neuron. Thus, training of neural network consists of finding the optimal set of weights and biases. ANNs are very popular among the scientific community as a pattern recognition tool especially for nonlinear regression problems. In fact, there is a common perception that if the data is preprocessed properly, a two layered neural network can model any complex practical data. Complex networks with large number of neuron layers can even model fine variation in training data but increases the risk of overtraining and loss of generalization. Unfortunately, no analytical method exists to determine optimal network topologies and one needs to rely on 'trial and error' methods based on the nature of training data for determining the optimum topology of a neural network.

The proposed system uses Back Propagation Neural Networks (BPNN) for modelling the relationship between pilot inputs and IMU responses. BPNN is a multilayer feed-forward network and uses error back propagation algorithm [17-18][21] for training. The neural network was trained to predict the IMU (Gyroscope) vibrational components resulting from pilot inputs obtained from the receiver module. A total of six indoor test flights were conducted in an enclosed hanger (at Davison Air Field), free of external disturbances (air wake and naturally imposed wind) for the collection of the data and training of the Neural Network. Three different $\mathrm{RC}$ helicopters (two flights with each helicopter) were used in these experiments and three separate sets of Neural Networks were trained to model the dynamics. The three helicopters used in the experiments were two TRex Pro 600E and one TRex ESP 600 and are referred to as 'Heli 1', 'Heli 2' and 'Heli 3', respectively throughout this paper. To obtain a versatile dataset of pilot inputs and helicopter responses, a variety of maneuvers (from extreme motion to steady hovering) were performed with these helicopters. During these experiments, the helicopters were 
flown at a significant height from the floor to avoid the 'ground effect' and impact of rotor wakes on helicopter dynamics.

\subsection{Neural Network Schema and Data Pre Processing}

RC helicopters, similar to every electro-mechanical system, require time to respond to pilot control inputs. Hence rather than instantaneous input, there is a need to consider the pilot input history for predicting the IMU output at any instant in time. Due to dimensionality issues, it is not possible to directly use the pilot input history as input vector. To reduce the dimensionality of the training data, pilot input history was approximated (within a fixed time window) to a linear function of time and line parameters were used to describe the pilot input history. For each pilot input channel (total of five), a history window of 43 samples (equivalent to $0.944 \mathrm{~s}$ of data, one cycle of cut-off frequency) were used and modeled with a line equation $y=m x+c$. Here $x$ is the sample index (time), $y$ is the concerned pilot input channel and $m$ and $c$ are the line parameters. The parameters $m$ and $c$ are calculated using the least square method as follows:

$$
\begin{gathered}
m=\frac{N \sum_{i=1}^{N} x_{i} y_{i}-\sum_{i=1}^{N} x_{i} \sum_{i=1}^{N} y_{i}}{N \sum_{i=1}^{N} x_{i}^{2}-\left(\sum_{i=1}^{N} x_{i}\right)^{2}} \\
\mathrm{c}=\frac{\sum_{i=1}^{N} x_{i}^{2} \cdot \sum_{i=1}^{N} y_{i}-\sum_{i=1}^{N} x_{i} y_{i} \sum_{i=1}^{N} x_{i}}{N \sum_{i=1}^{N} x_{i}^{2}-\left(\sum_{i=1}^{N} x_{i}\right)^{2}}
\end{gathered}
$$

$N$ is the number of samples in the window which is 43 in our case. To account for non-linearity in the modelling of pilot inputs, the sum of the absolute errors $(e)$ as a third parameter modeled pilot input data and was calculated as follows:

$$
e=\sum_{i=1}^{N}\left|y_{i}-\left(m x_{i}+c\right)\right|
$$


Hence for every 43 sample window in each pilot input data channel, three parameters $\{m, c, e\}$ were obtained. Since there are five data channels, the pilot input data was characterized by 15 parameters. As discussed in Section 2, the angular rate measurements depend on the immediate predecessor measurement. In addition to pilot input data, the latest previous measurements from the three gyroscope channels were also fed into the neural network. It is worth noting that despite of possible high frequency noise, no low pass filter was applied on the data to avoid any temporal interdependence in data due to the filtering process.

In the WTS, four channels of gyroscope data were predicted using four different neural networks. The first three channels were three Cartesian components of filtered gyroscope data and the fourth one was the local standard deviation of the magnitude of Gyroscope data. Therefore, each channel of the IMU data was predicted using a separate Back Propagation Neural Network (BPNN). Four layered BPNN with two hidden layers were used for modeling the Gyroscope data from Pilot RC signals. Each BPNN took an 18-dimensional input vector (i.e., 15 pilot data and 3 channels of previous gyroscope measurement sample) to predict a 1dimensional output (i.e., current sample of the specific channel of Gyroscope data). Before feeding the IMU data (Gyroscope) and the pilot input PWM signals to the neural networks, all the data was normalized to zero mean and unit standard deviation to avoid any numerical issues during the training phase. The actual mean and standard deviation of the training data was stored in computer for scaling and rescaling the telemetry data during the testing phase of the neural networks.

Fig. 5 shows the general topology of the neural network used in the system. The size of the input and the output layer as determined by the dimensionality of input and output data that was 15 and 1 node respectively. The network topology for the hidden layers was selected using 'trial 
and error' method. The number of nodes was varied from 5 to 14 for the first hidden layer and from 2 to 8 for the second hidden layer and the topology giving the highest prediction accuracy was selected.

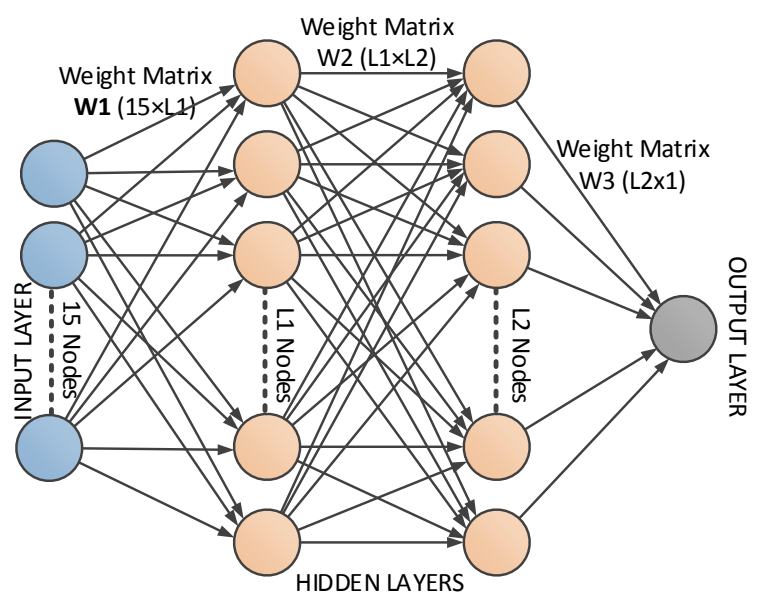

Figure 5 Topology of Neural Networks used in WTS

\subsection{Neural Network Training and Performance}

Back-Propagation Neural Network training involves assigning initial weights randomly; thus, it is possible that the training accuracy obtained from a particular topology is not the 'best' set of chosen parameters. This is due to the fact that the BPNN training process is analogous to gradient descent algorithm and is susceptible to local minima in the weight-error space while obtaining optimum weights. To overcome this limitation, we trained the network 30 times for each topology and considered the 'overall best network' for the IMU prediction in the 'trial and error' procedure. Table 1 shows the network topologies finally selected for the twelve BPNNs. $10 \%$ the total data was used for training and a 10 -fold cross validation [22] was applied to prevent overtraining of the network. 
Table 1 BPNN Network Topologies

\begin{tabular}{c|cc|cc|cc|cc}
\hline \hline \multirow{3}{*}{ Helicopter } & \multicolumn{8}{c}{ Neural Network } \\
\cline { 2 - 9 } & \multicolumn{2}{c|}{ Net1 } & \multicolumn{2}{c}{ Net2 } & \multicolumn{2}{c}{ Net3 } & \multicolumn{2}{c}{ Net4 } \\
\cline { 2 - 9 } & L1 & L2 & L1 & L2 & L1 & L2 & L1 & L2 \\
\hline Heli 1 & 7 & 6 & 7 & 4 & 8 & 4 & 10 & 4 \\
Heli 2 & 7 & 4 & 8 & 6 & 7 & 4 & 11 & 4 \\
Heli 3 & 7 & 3 & 7 & 5 & 9 & 3 & 11 & 3 \\
\hline \hline
\end{tabular}

After obtaining the optimum sets of neutral networks for the three helicopters, the neural network sets were tested on the remaining 90\% data. Fig. 6 shows five performance subplots of the four neural networks in the estimation of the four Gyroscope data channels along with pilot inputs for 'Heli 1'. In these subplots, the red colored plots show data obtained from the sensor measurements whereas the blue plots show the predictions from BPNN using pilot inputs.

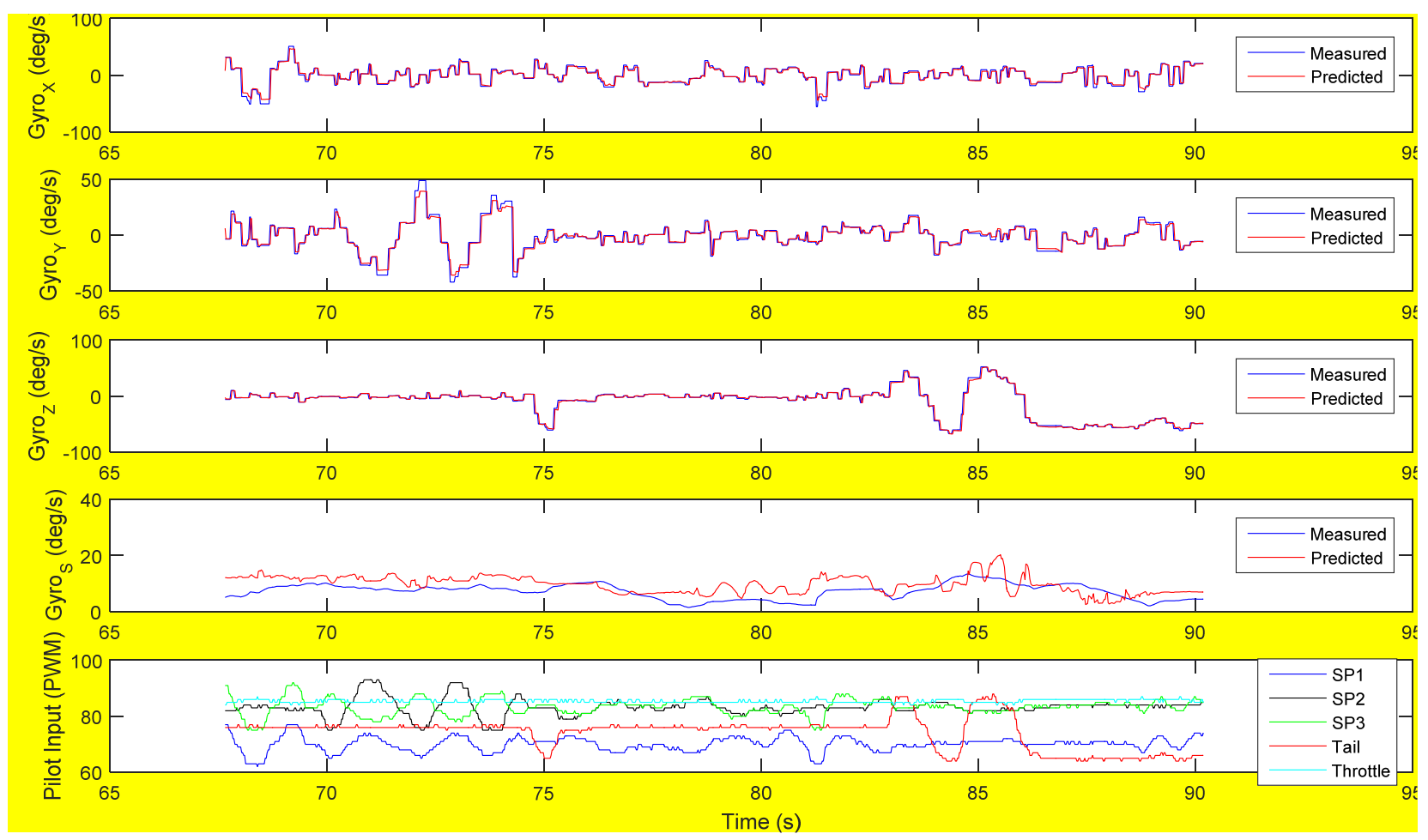

Figure 6 Prediction by neural networks corresponding to Heli 1 (showing 25 seconds of data) 
For quantitative analysis of predicting the results, histograms were plotted in Fig. 7 showing the prediction error distribution of the neural networks trained for all three helicopters. In addition to this, RMS prediction error for the neural networks is also shown in Table 2. The RMS values of the prediction errors varied between 2 and $7 \mathrm{deg} / \mathrm{s}$, which is reasonably good for a noisy platform such as an RC helicopter.

Table 2 BPNN Prediction RMS Error (deg/s)

\begin{tabular}{c|c|c|c|c}
\hline \multirow{2}{*}{ Helicopter } & \multicolumn{4}{c}{ Neural Network } \\
\cline { 2 - 5 } & Net1 & Net2 & Net3 & Net4 \\
\hline Heli 1 & 8.28 & 5.93 & 5.10 & 3.44 \\
Heli 2 & 3.84 & 6.81 & 2.93 & 2.13 \\
Heli 3 & 7.61 & 17.16 & 5.62 & 3.55 \\
\hline \hline
\end{tabular}



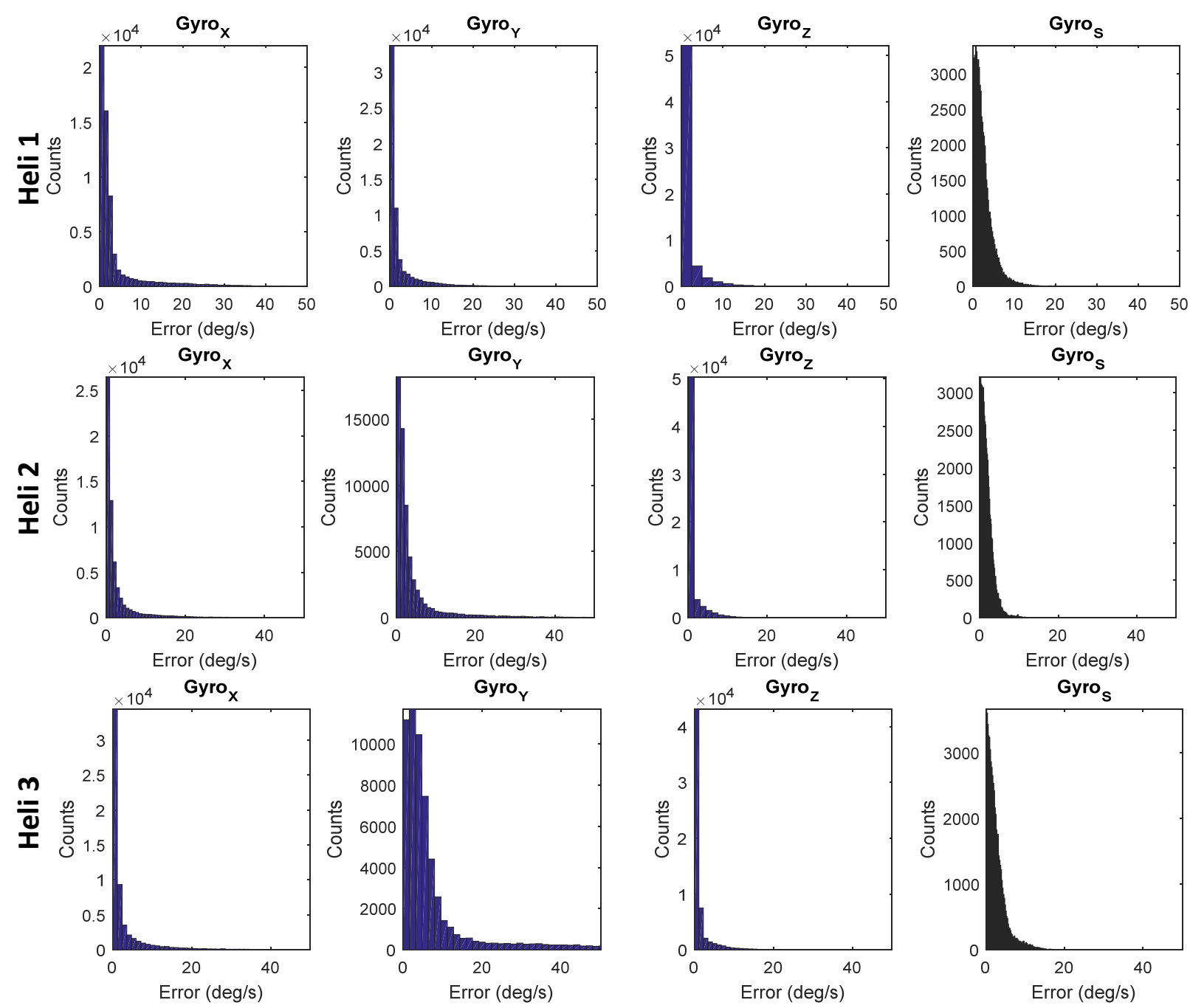

Figure 7 Error distributions for the prediction by the neural networks corresponding to the three helicopters

\subsection{Air wake estimation with pilot input compensation}

As mentioned previously, WTS was tested in a naval application for ship/air wake pattern detection for determining safe launch and recovery envelopes on naval vessels. In such an application, it's important to find flight paths with minimum air wake intensity. Air wake intensity is inferred to be proportional to angular speed of tilting. Therefore, it is advantageous to use radial component of Gyroscope data rather than three Cartesian components to decrease the 
computational burden. In the proposed system, the gyroscope data was converted to a spherical coordinate system and the absolute magnitude (radial component) was used to represent air wake patterns. If $\left\{\omega_{x}, \omega_{y}, \omega_{z}\right\}$ is the filtered angular velocity $\left(\omega_{f}\right)$ of the helicopter in Cartesian coordinate system measured from the gyroscope and $\left\{\omega_{x}^{\prime}, \omega_{y}^{\prime}, \omega_{z}^{\prime}\right\}$ is the angular velocity $\left(\omega_{f}^{\prime}\right)$ determined from the Neural Network, then the radial component of the net angular velocity $\left(\omega_{r}\right)$ due to air wake is obtained as follows:

$$
\omega_{r}=\left\|\omega_{f}-\omega_{f}^{\prime}\right\| .
$$

It is not only the tilting (angular) velocity that accounts for air wake related problems but also the fluctuation of angular velocity (i.e., angular acceleration). Thus, high air wake zones are characterized by both high angular velocity and angular acceleration. This fluctuation in angular velocity will appear as a peak in the gyroscope absolute magnitude (radial) component as well as a peak in the local standard deviation of the gyroscope radial component. In this paper, we don't consider the direction of air wakes, the radial component (magnitude) of gyroscope is sufficient for analysis of air wake patterns. Under the effects of air wake, the helicopter is supposed to experience high angular accelerations. Such changes in angular velocities can be measured by local standard deviation of the gyroscope data (radial component). The local standard deviation was calculated by applying standard deviation filter with a window size of $0.944 \mathrm{~s}$ (L samples). The $\mathrm{i}^{\text {th }}$ sample of the local standard deviation $\left(\omega_{s}\right)$ of the radial component of the raw gyroscope data $(\omega)$ is calculated as follows:

$$
\omega_{s}(i)=S D(\omega(i+d)) ; i, d \in[1, N] ; d \in[-L / 2, L / 2] \text {. }
$$

where $N$ is the total number of samples in $\omega$ and $L$ is the length of the filter window. 
A simultaneous rise is expected in the filtered gyroscope data and its standard deviation data. The product of both waveforms (point-to-point multiplication) is used to represent air wake intensity. Furthermore, the standard deviation $\left(\omega_{s}\right)$ obtained from the raw gyroscope data also contains the components of vibrations arising from pilot inputs. Therefore, there is a need to compensate for it while predicting air wake. For convenience, the generated waveform is referred to as the 'Air Wake data' $\left(A_{\omega}\right)$ :

$$
A_{\omega}(i)=\left(\omega_{s}(i)-\omega_{s}(i)^{\prime}\right) \times \omega_{r}(i) ; \quad i \in[1, N] .
$$

Where $\omega_{s}^{\prime}$ is the standard deviation of the gyro data predicted from the Neural Network.

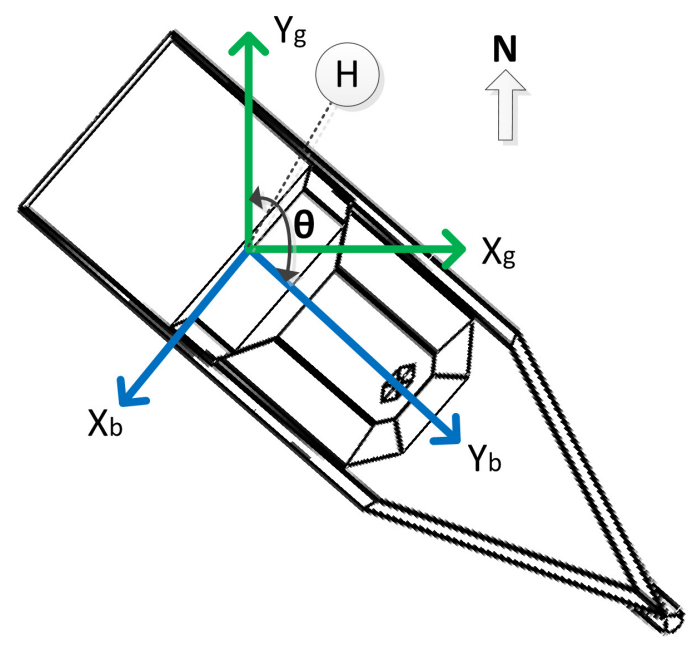

Figure 8 Coordinate system on the ship for localizing the helicopter

\section{AIR WAKE PATTERN ESTIMATION}

In order to detect air wake patterns, it is important to accurately locate the transmitter carrier in the receiver module's frame of reference. As mentioned earlier, the proposed system uses GPS and IMU to locate the transmitter. The orientation of the receiver module was estimated by using the implementation of the tilt compensated compass [23] using the accelerometer and magnetometer in the receiver module. As shown in Fig. 8, the heading angle of the boat (receiver 
module) $\theta$ is in the geographical frame of reference. If $\left\{\lambda_{b}, \phi_{b}\right\}$ and $\left\{\lambda_{h}, \phi_{h}\right\}$ are the geographical coordinates of the boat and the helicopter, respectively, then the relative position of helicopter w.r.t the boat in Cartesian coordinates is given by:

$$
\left[\begin{array}{l}
x_{h} \\
y_{h}
\end{array}\right]_{b}=\left[\begin{array}{cc}
\cos \theta & \sin \theta \\
-\sin \theta & \cos \theta
\end{array}\right][S]\left[\begin{array}{l}
\lambda_{h}-\lambda_{b} \\
\phi_{h}-\phi_{b}
\end{array}\right] ; \quad[S]=\left[\begin{array}{cc}
11.1 & 0 \\
0 & 8.6
\end{array}\right] \times 10^{4}\left(m /^{\circ}\right) .
$$

To test the system's performance in an actual air wake measurement, multiple flight operations were conducted in the Chesapeake Bay, MD over a period of one year. The test flights were conducted at a constant height and heading direction of the helicopter. The wind conditions were maintained constant within the specified limits. Air wake status intensity along with the helicopter location (transmitter carrier) was presented and recorded in real-time through GUI software developed in National Instruments LabVIEW ${ }^{\mathrm{TM}}$. Fig 9 shows the GUI developed to interface with the hardware and for processing the data in real-time. 


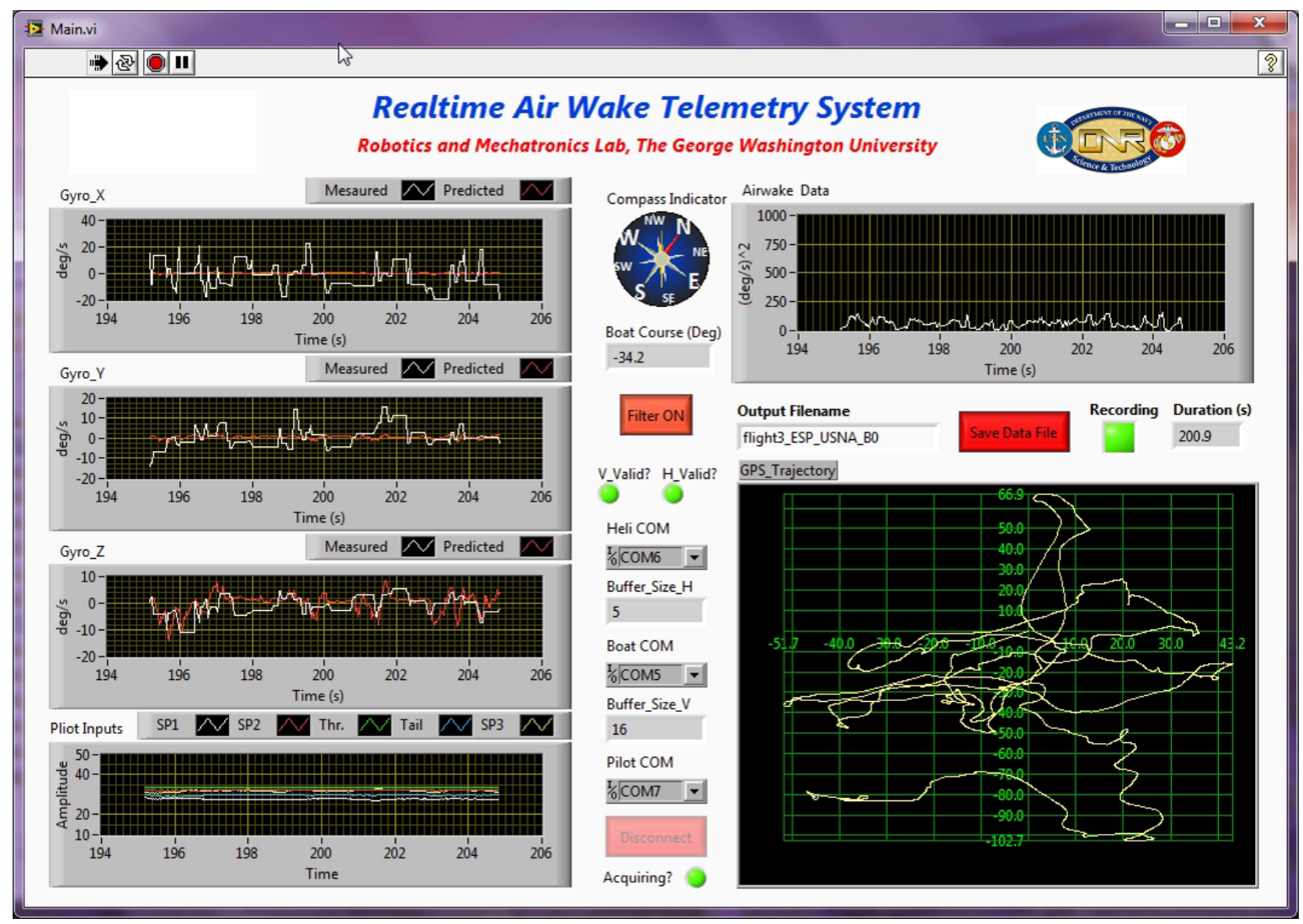

Figure 9 GUI for proposed wireless telemetry system

For final estimation of air wake pattern, the air wake data as obtained through (7) is overlaid on the trajectory of the helicopter in the form of spatial distribution plots. In these plots, the color on the helicopter trajectory represents the air wake magnitude after pilot input compensation. Fig. 10 shows sample ship air wake pattern obtained in a test flight of relative wind angle (beta angle) $0^{\circ}$ and $15^{\circ}$. A black dotted curve shows the region of high air wake with respect to the flight deck. A high perturbation is detected along the outer regions of the trajectory as the WTS measured differential wind velocities. This is in accordance with the wind conditions and related numerical simulations as it is symmetric about the centerline of the helicopter trajectory [6,7]. In this figure, the high air wake region as marked by the dotted curve is tilted towards the right relative to the stern of the ship, which again correlates this result with numerical simulations $[6,7]$. 

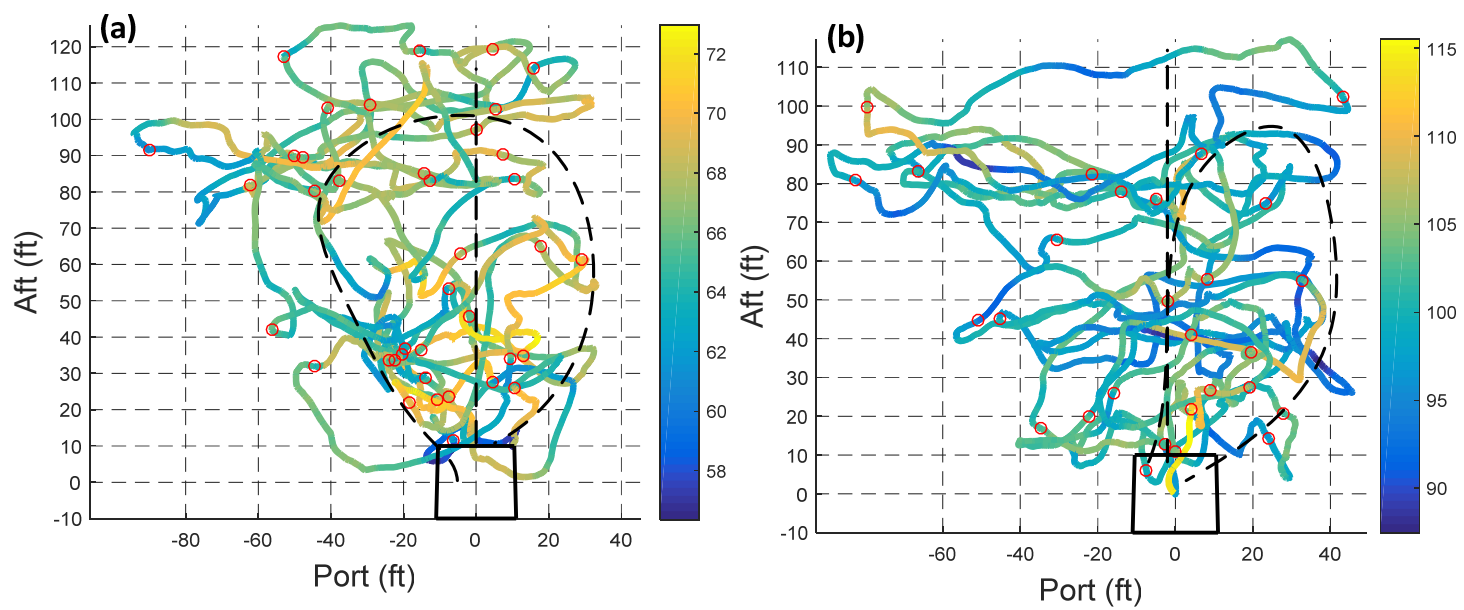

Figure 10 Ship air wake distribution for: (a) test flight with beta angle of $0^{\circ}$, (b) test flight with beta angle of $15^{\circ}$

\section{CONCLUSION AND FUTURE WORK}

This paper proposes a new technique that accurately detects turbulence patterns in large, open spaces where conventional instrumentation cannot be used. Turbulence patterns were mapped out relative to a ship using the WTS mounted on an RC helicopter and a trained neural network eliminated pilot induced vibrations from measured data with good accuracy. This new means of air wake pattern detection will bring about enhanced flight research capabilities, and improvements for aerial vehicle design and operational safety analysis in the Aerospace Industry. Although this paper presents an effective tool for determining air wake distribution pattern, the applications of this system can be broadened.

Future work includes use of sensitive Inertial Navigation System to measure translational velocities and accelerations of the helicopter with pilot input compensation. Since the magnitude and the direction of the helicopter's velocity is the same as that of air wakes, this upgrade will enable the system to determine the thrust directions and Cartesian components of turbulence forces. In this paper, BPNNs were used in modeling the contribution of pilot input in helicopter's dynamics. The authors also plan on implementing Bayesian Regression methods to inherently 
model data and noise separately and hence provide better prediction accuracy. To convert the air wake measurements from IMU units to actual air velocity units, the authors plan to calibrate the proposed system in a closed environment (hangar). Fig. 11 shows the experimental setup to be used for calibration. During the calibration process the instrumentation system will be retrofitted with two lightweight ultrasonic anemometers and used to directly measure a known wind pattern generated by fans. The authors plan to further extend this work to develop autonomous flight control system capable of rejecting external disturbances.

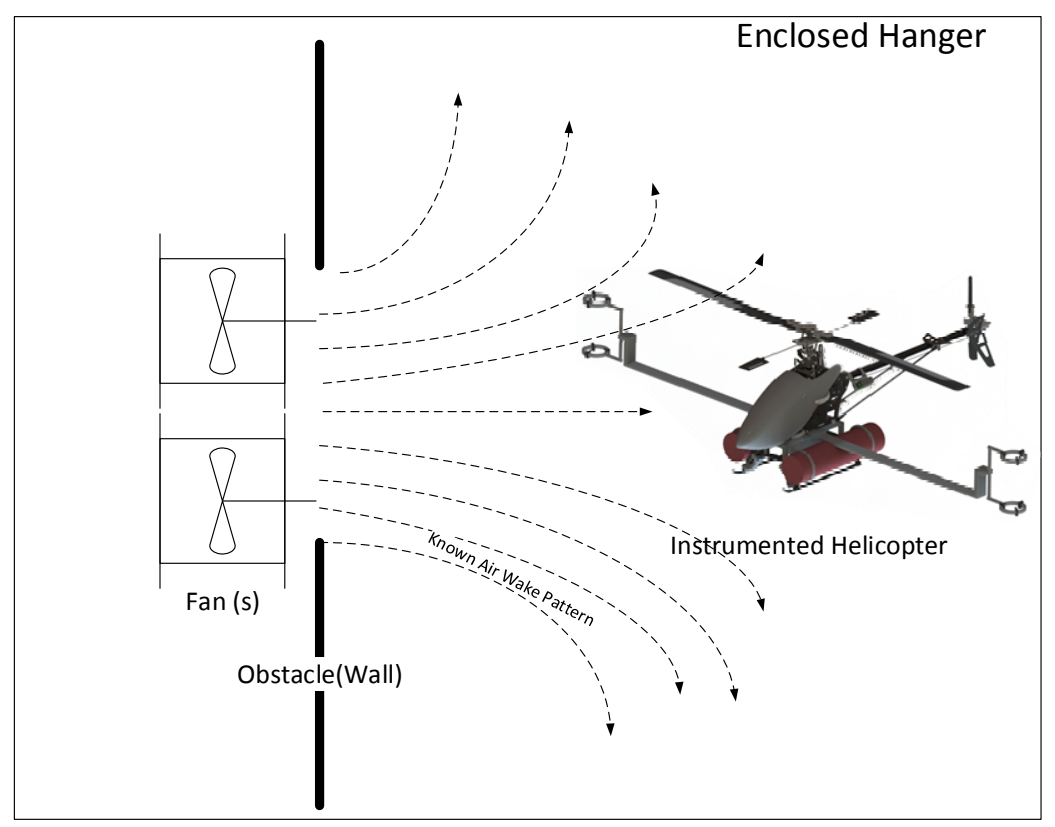

Figure 11 Calibration of the Wireless Telemetry System

\section{Acknowledgements}

This research is funded by the Office of Naval Research. Program Officer is Mr. John Kinzer (Code 351). 


\section{References}

[1] Maldonado F.J., Oonk S. and Politopoulos T., 2013, "Enhancing vibration analysis by embedded sensor data validation technologies," IEEE Instrumentation \& Measurement Magazine, vol.16, no.4, pp. 50-60.

[2] Cooper R.D. and Tulin M.P., 1955, "Turbulence measurements with the hot-wire anemometer", NATO, Advisory Group for Aeronautical Research and Development, [Online]. Available: http://ftp.rta.nato.int/public/PubFullText/AGARD/AG/AGARD-AG-12/AGARDAG-12.pdf.

[3] Mann J., Cariou J.P., Courtney M.S., Parmentier R., Mikkelsen T., Wagner R., Lindelöw P., Sjöholm M. and Enevoldsen K., 2008, "Comparison of 3D turbulence measurements using three staring wind lidars and a sonic anemometer," IOP Conference Series: Earth and Environmental Science. vol. 1. no. 1. IOP Publishing, DTU, Denmark, 23-25 June, 2008.

[4] Kumar A., Ben-Tzvi, P., Snyder, M.R. and Saab, W., "Instrumentation System for Ship Airwake Measurement", Proceedings of the 2013 IEEE International Symposium on Robotic and Sensors Environments (ROSE 2013), Washington, DC, Oct. 21-23, 2013

[5] Miklosovic D.S., Kang H.S. and Snyder M.R., "Ship Air Wake Wind Tunnel Test Results," 29th AIAA Applied Aerodynamics Conf., Honolulu, Hawaii, June 2011.

[6] Snyder M.R., Kang H.S. and Burks J.S., "Comparison of Experimental and Computational Ship Air Wakes for a Naval Research Vessel," 30th Applied Aerodynamics Conf., New Orleans, Louisiana, June 2012.

[7] Snyder M.R., Kumar A., Ben-Tzvi P. and Kang H.S., "Validation of Computational Ship Air Wakes for a Naval Research Vessel," 51st AIAA Aerospace Sciences Meeting, AIAA, Grapevine, Texas, 7-10 Jan. 2013. 
[8] Brownell C.J., Luznik L., Snyder M.R. and Kang H.S., Wilkinson C.H., 2012, "In situ air velocity measurements in the near wake of a ship superstructure," AIAA Journal Aircraft, vol. 49, no. 5, pp. 1440-1450.

[9] Snyder M.R., Kang H.S. and Brownell C.J., "Validation of Ship Air Wake Simulations and Investigation of Ship Air Wake Impact on Rotary Wing Aircraft", Naval Engineers Journal, vol. 125, no. 1, March 2013.

[10] X. Lei and P. Lu, "The Adaptive Radial Basis Function Neural Network for Small-Rotary Wing Unmanned Aircraft,” IEEE Transactions Industrial Electronics, 61(9), 2014, pp.48084815.

[11] Liu H., Lu G, and Zhong Y., "Robust LQR Attitude Control of a 3-DOF Laboratory Helicopter for Aggressive,” IEEE Trans. Ind. Electron., 60(10), 2013, pp.4627-4636.

[12] X. Niu et al., "Locomotion Learning for an Anguilliform Robotic Fish Using Central Pattern Generator Approach,” IEEE Trans. Ind. Electron, 61(9), 2014, pp.4780-4787.

[13] A. Kumar, P. Ben-Tzvi and M.R. Snyder, "UAV-Based Wireless Telemetry System for the Estimation of Ship Air Wake Patterns", Proceedings of the 2015 ASME/IEEE International Conference on Mechatronic and Embedded Systems and Applications (MESA), IDETC 2015, Boston, Massachusetts, Aug. 2-5 2015

[14] G.D. Padfield, "Helicopter Flight Dynamics", Blackwell Publishing, Oxford, Second Edition, 2007

[15] R. Rojas, Neural Networks - A Systematic Introduction, Springer-Verlag, Berlin, NewYork, 1996, pp 151-184.

[16] Haykin S., Neural Networks: A Comprehensive Foundation, Prentice-Hall, Englewood Cliffs, NJ, 1999. 
[17] Simpson P.K., Artificial Neural Systems, Pergmon Press, Elmsford, New York, 1989.

[18] Karnin E.D., "A simple procedure for pruning back propagation trained neural networks", IEEE Trans. Neural Networks, 1(2), 1990, pp. 239-242.

[19] J.S. Wang and F.C. Chuang, "An Accelerometer-Based Digital Pen with a Trajectory Recognition Algorithm for Handwritten Digit and Gesture Recognition,” IEEE Trans. Ind. Electron, 59(7), 2012, pp.2998-3007.

[20] Estanjini R.M., Lin Y., Li K., Guo D. and Paschalidis I.C., "Optimizing Warehouse Forklift Dispatching Using a Sensor Network and Stochastic Learning," IEEE Trans. Ind. Electron, 7(3), 2011, pp.476-486.

[21] B. Widrow, and S.D. Sterns, Adaptive Signal Processing, New York, Prentice-Hall, 1985.

[22] R. Kohavi, "A study of cross-validation and bootstrap for accuracy estimation and model selection". Proc. 14th International Joint Conf. Artificial Intelligence, 1995, 2(12): pp. 11371143.

[23] T. Ozyagcilar, "Implementing a Tilt-Compensated eCompass using Accelerometer and Magnetometer Sensors", Free Scale Application note, [Online]. Available: http://freescale.com.hk/files/sensors/doc/app_note/AN4248.pdf 